Revue d'histoire de l'Amérique française

REVUE D.HISTOIRE DE L'AMÉRIQUE FRANÇAISE

\title{
Le Bas-Canada
}

\section{Une étape importante dans l'oeuvre de Fernand Ouellet}

\section{Pierre Tousignant}

Volume 34, numéro 3, décembre 1980

URI : https://id.erudit.org/iderudit/303881ar

DOI : https://doi.org/10.7202/303881ar

Aller au sommaire du numéro

\section{Éditeur(s)}

Institut d'histoire de l'Amérique française

\section{ISSN}

0035-2357 (imprimé)

1492-1383 (numérique)

Découvrir la revue

\section{Citer cette note}

Tousignant, P. (1980). Le Bas-Canada : une étape importante dans l'oeuvre de Fernand Ouellet. Revue d'histoire de l'Amérique française, 34(3), 415-436.

https://doi.org/10.7202/303881ar d'utilisation que vous pouvez consulter en ligne.

https://apropos.erudit.org/fr/usagers/politique-dutilisation/ 


\title{
NOTE CRITIQUE \\ LE BAS-CANADA*: \\ UNE ÉTAPE IMPORTANTE DANS \\ L'OEUVRE DE FERNAND OUELLET
}

\author{
PIERRE TOUSIGNANT \\ Département d'histoire \\ Université de Montréal
}

C'est à juste titre que Michael D. Behiels ${ }^{1}$ a qualifié cet ouvrage de «seconde contribution majeure» de Fernand Ouellet depuis la parution, en 1966, de son imposante Histoire économique et sociale du Québec, 1760-1850. Et il importe de savoir dès l'abord que c'est sur la lancée de sa première grande et ambitieuse entreprise que l'auteur a élaboré sa reconstitution de l'histoire du Bas-Canada. Il a voulu, explique-t-il dans sa préface, situer son analyse dans une même «perspective globale» en tenant compte «de l'interaction de multiples forces» qui agissent sur le développement d'une société, mais en mettant l'accent, cette fois, sur «les mutations sociales et politiques» et en réservant «une attention spéciale» aux diverses composantes de «la structure socio-ethnique». La différenciation entre ces deux ouvrages est cependant beaucoup plus prononcée que ne le laisse entendre le préfacier. D'une orientation principalement axée sur l'évolution et les transformations économiques du Québec, l'auteur en est venu à centrer son analyse sur «l'émergence d'une crise qui peu à peu empoigne toute la société et se dénoue au cours d'une aventure révolutionnaire».

D'emblée l'auteur nous avise qu'il s'en est tenu au même «schéma interprétatif» qu'il avait "construit» pour son magnum opus des années 1960, ses recherches ultérieures lui en ayant confirmé «la validité». Ainsi prévenu, le lecteur ne saurait se méprendre sur le sens de sa déclaration: «Notre livre tient compte [d'une] historiographie en constante évolution." Il s'en faut et de beaucoup pour que Fernand Ouellet admette avoir été influencé par les apports des quelques historiens jugés dignes

* Fernand Ouellet, Le Bas-Canada, 1791-1840 - Changements structuraux et crise, Cahier d'histoire de l'Université d'Ottawa no 6 (Ottawa, Editions de l'Université d'Ottawa, 1976; 2e édition, 1980), 541 p.

1 Compte rendu critique paru dans la CHR, LIX, 2 (juin 1978): 226-229.

RHAF, vol. 34, no 3, décembre 1980

[415] 
d'être mentionnés dans sa préface. On ne doit donc pas s'attendre à le voir reconnaître "la validité» de «schémas interprétatifs» autres que le sien et encore moins avouer y avoir fait des emprunts. L'auteur appartient à cette catégorie d'intellectuels qui, pour l'essentiel, n'empruntent qu'à eux-mêmes.

Pour s'approprier Clio, l'auteur a privilégié deux approches: la voie de l'analyse «quantitative» par méthode cumulative de données chiffrées de toutes sortes et de statistiques surtout descriptives et la voie «sociologique» réduite au modèle weberien d'une typologie différentielle des mentalités. La combinaison de ces deux voies lui a valu d'être porté aux nues, notamment dans les milieux universitaires anglophones ${ }^{2}$. Sous le couvert d'un impressionnant appareillage à caractère réputé scientifique, le Fernand Ouellet de l'Histoire économique et sociale du Québec faisait écho aux vieux préjugés de l'historiographie canadienne-anglaise tout imprégnée de l'idéologie de supériorité de la race anglo-saxonne. À défaut de changer de «mentalité» et d'adopter l'esprit d'entreprise capitaliste des conquérants, annonçait-il en introduction de son ouvrage, les Canadiens français risquaient de demeurer, selon l'expression de lord Durham, "an old and stationary society, in a new and progressive world» - celui de ia colonisation britannique.

Fort heureusement, ce genre d'approche axée sur un évolutionnisme unilinéaire qui justifiait la notion désormais dépassée du «retard des mentalités» n'apparaît plus au premier plan dans Le Bas-Canada. C'est une notable amélioration qui mérite d'autant plus d'être soulignée que l'auteur adopte à ce sujet le ton de la discrétion. On ne peut cependant en dire autant de sa thèse d'un Canada français rural s'enlisant dans une "crise agricole» dès le début du dix-neuvième siècle. Cette question fort controversée ayant fait l'objet d'un sérieux «examen critique» par les professeurs Gilles Paquet et Jean-Pierre Wallot ${ }^{3}$, il serait superflu de prolonger ici le débat. Qu'il suffise de signaler la façon scolastique avec laquelle le professeur Ouellet récuse leur «remise en question» qualifiée d' "antithèse» en faisant appel à de bonnes vieilles notions de logique aristotélicienne. Tant que ne sera pas démontrée la fausseté de la thèse, argumente-t-il, celle-ci demeurera vraie, à l'exclusion de toute autre hypothèse. Ainsi nous enseignait-on jadis à raisonner sur les preuves de l'existence de Dieu.

${ }^{2}$ Le compte rendu critique de Michael D. Behiels témoigne de l'étonnante persistance de ce courant d'admiration sans borne: "Le Bas-Canada achieves and surpasses the exceedingly high standards set by his Histoire économique et sociale du Québec... M. Ouellet maintains the same 'quantitative' methodological approach - refined and reinforced by a vast assortment of statistical data - to construct an impregnable foundation for his interpretation of the socio-political crises..."

3 Voir leur étude de base intitulée «Crise agricole et tensions socio-ethniques dans le Bas-Canada, 1802-1812: éléments pour une ré-interprétation", RHAF, 26, 2 (septembre 1972): $185-237$. 
Rappelons brièvement que la thèse ouelletienne de «l'émergence d'une crise agricole» dès la première décennie du dix-neuvième siècle repose essentiellement sur deux séries de données statistiques: l'une permettant de reconstituer la fluctuante courbe des exportations de blé et de farine; l'autre, fort incomplète, fournissant des renseignements partiels sur l'évolution des rendements de la dîme en blé dans les paroisses du Bas-Canada. Dans les deux cas, l'auteur relie l'interprétation de ces données à la «question centrale» de la production dont «l'irréversible déclin» se produisit, selon lui, après «la grande date tournante» de 1802, marquant ainsi le début d'une "crise» qui, de décennie en décennie, alla s'aggravant pour finalement constituer la toile de fond de la période trouble des années 1830-1840.

S'il n'y a pas lieu de débattre ici «la validité» de cette thèse ni d'en récuser la possibilité d'application après mais non avant 1815 , il importe au moins d'en connaître les failles «scientifiques» dans ses fondements méthodologiques eux-mêmes.

Les années 1801 et 1802 , du point de vue des exportations de blé et de farine, permirent d'atteindre un niveau record qui demeurera inégalé: soit un peu plus de 1800000 minots, ce qui représentait en moyenne le double des meilleures années de la période 1791-1812. Or, plutôt que de considérer statistiquement 1801 et 1802 comme des cas d'exception ${ }^{4}$, l'auteur se donne beau jeu de montrer une situation se dégradant dès 1803 en utilisant, pour fins de comparaison de moyennes annuelles, deux blocs décennaux dont l'un inclut ces deux années exceptionnelles: 17931802 vs 1803-1812 (page 179). Le choix de cette coupure n'est évidemment pas innocent et comporte une bonne part d'arbitraire qui ne trouve son ultime justification qu'en fonction de la thèse de l'auteur. La situation apparaîtrait fort différente en déplaçant, ni plus ni moins arbitrairement, les années 1801 et 1802 d'un bloc à l'autre pour comparer les moyennes annuelles des décennies 1791-1800 vs 1801-1810.

La deuxième série de données statistiques n'échappe pas non plus à cet arrangement pro domo (tableau de la page 181 où une correction s'impose: lire 85 et non 165 paroisses). Il allait de soi que, dans les années de bonnes récoltes, la masse paysanne fît montre de plus de générosité envers ses curés. Ainsi l'année 1802 fut si exceptionnelle à cet égard que dans vingt-trois des vingt-six paroisses visitées par l'évêque Denault, la moyenne des contributions en blé par communiant s'éleva à un demiminot et plus. Il n'en fallait pas davantage pour que l'auteur prétende avoir découvert la preuve d'une "véritable cassure» dans le rythme de production après 1802. Mais ici encore, il suffit d'opérer un déplacement

${ }^{4}$ La soudaine et vertigineuse montée du prix du blé en Angleterre en 1800 et 1801 créa une occasion exceptionnelle pour les marchands du Bas-Canada de maximiser leur profit en vidant les greniers des habitants. 
pour faire ressortir la fragilité de la construction ouelletienne. Illustrons le rendement moyen de la dîme en blé par les deux tableaux suivants: A) incluant les vingt-six paroisses visitées en 1802; B) en les excluant.

\begin{tabular}{|c|c|c|c|}
\hline décennies & moins de $1 / 2$ minot & $\begin{array}{l}\text { de } 1 / 2 \text { à } .99 \text { minot } \\
-\mathrm{A}-\end{array}$ & un minot et plus \\
\hline $\begin{array}{l}\overline{1791-1800} \\
1801-1810\end{array}$ & $\begin{array}{l}23 / 91=25,3 \% \\
41 / 159=25,8 \%\end{array}$ & $\begin{array}{c}53 / 91=58,2 \% \\
100 / 159=62,9 \% \\
-\mathrm{B}-\end{array}$ & $\begin{array}{l}15 / 91=16,5 \% \\
18 / 159=11,3 \%\end{array}$ \\
\hline $\begin{array}{l}1791-1800 \\
1801-1810\end{array}$ & $\begin{array}{l}23 / 91=25,3 \% \\
38 / 133=28,6 \%\end{array}$ & $\begin{array}{l}53 / 91=58,2 \% \\
87 / 133=65,4 \%\end{array}$ & $\begin{aligned} 15 / 91 & =16,5 \% \\
8 / 133 & =6,0 \%\end{aligned}$ \\
\hline
\end{tabular}

Dans les deux cas, on se trouve en présence de plus de $70 \%$ de la masse paysanne contribuant pour un demi-minot et plus. Ce n'est qu'après 1815 que sera repéré un mouvement de bascule entraînant, au cours de la troisième décennie du dix-neuvième siècle, plus de $70 \%$ de la masse paysanne dans la catégorie des «moins d'un demi-minot».

La «crise agricole» préoccupe à ce point Fernand Ouellet qu'il en cherche des signes partout. D'une apostrophe lancée par le journal Le Canadien, au moment de l'affaire de la frégate Chesapeake en 1807, contre l'immigration au Bas-Canada de "Yankés, Austrogoths, Vandales...», l'auteur en conclut à un "drame» pour les milieux ruraux qu'il voit «déjà aux prises avec le problème du surpeuplement des seigneuries» (p. 116). Il va même jusqu'à reprendre un témoignage de John Neilson exposant, en 1823, devant le Comité de la Chambre que le problème de la disponibilité des terres dans le cadre de la zone seigneuriale avait commencé à se poser sérieusement au lendemain de la guerre de 1812-1814. Tout en reconnaissant la validité du diagnostic posé par l'éditeur de la Gazette de Québec (rareté réelle ou artificiellement créée, soit par le refus des seigneurs de concéder des terres réservées à l'exploitation forestière, soit par une augmentation abusive du taux des rentes ou par l'imposition d'onéreuses conditions de concessions), l'auteur juge que Neilson «situe une décennie trop tard» l'origine de ce surpeuplement (p. 221)!

Sur le sort de la paysannerie, l'auteur s'exprime avec des accents de sympathie auxquels ne nous avait pas habitués son Histoire économique et sociale du Québec. Le Fernand Ouellet des années ' 60 n'était guère tendre à l'endroit des «habitants» du Bas-Canada qu'il rendait pratiquement responsables des maux et des malheurs qu'ils avaient éprouvés durant près d'un demi-siècle de crise agricole. Non seulement reprochaitil à la masse rurale canadienne-française son enlisement dans la routine, son insensibilité à l'égard de toute forme de progrès et son refus de tout 
changement tant dans les méthodes que dans les techniques de culture, mais il la blâmait d'avoir voulu échapper à la famine et à la misère en délaissant la production du blé au profit de celle de la pomme de terre pourtant la seule garantie de subsistance pour une population rurale devenue trop nombreuse dans le cadre de la zone seigneuriale. Il attribuait même l'appauvrissement progressif de la paysannerie à «sa propension aux dépenses somptuaires et aux investissements improductifs... en dépit des circonstances malheureuses qui l'accablaient» (Histoire économique et sociale, p. 456).

Le Fernand Ouellet du Bas-Canada présente une meilleure approximation de la réalité historique. Qu'on en juge par le passage suivant:

La chute de la production du blé, la disparition progressive des surplus et la lenteur de l'adaptation, quand ce n'est pas le simple refus de l'ajustement, compriment l'existence paysanne. On peut même se demander si celle-ci n'avait pas toujours été associée d'une façon générale avec la misère. Dans ce cas, il faudrait ne voir dans tous ces cheminements qu'un approfondissement d'une condition déjà marquée par une pauvreté permanente (p. 192).

Que de chemin parcouru pour en arriver à s'exprimer de la sorte; cela paraît d'autant plus remarquable pour qui connaît la profondeur du fossé qui séparait Fernand Ouellet de l'école néo-nationaliste de l'Université de Montréal et de son maître à penser, le professeur Maurice Séguin. Comment, en effet, ne pas évoquer le nom de ce dernier lorsqu'on lit dans Le Bas-Canada que «l'infériorité économique des Canadiens français», sous l'effet de la domination britannique, avait acquis en 1791 «un caractère presque inexorable» (p. 63) et que la condition de la masse paysanne portait le sceau d'une «pauvreté permanente»? Il y a déjà plus de trente ans, le professeur Séguin, dans sa thèse de doctorat, soutenait que la Conquête n'avait laissé en partage au peuple conquis que l'exploitation de ses terres, ce qui constituait «un bien piètre moyen d'essor économique» dans un Québec où «il n'y avait pas de place pour une grande agriculture rémunératrice ${ }^{5}$.

Que la terre québécoise fut «peu rémunératrice» avant de devenir «improductive», le professeur Ouellet semble maintenant en convenir et, dans cette perspective qui tient compte de la réalité des limites à l'enrichissement collectif de la "nation canadienne», il devient possible de faire la part des responsabilités. Il ne saurait plus être question de porter au seul compte d'une différence de mentalités l'appauvrissement progressif de la majeure partie de la paysannerie. Reconnaître qu'avec ou sans l'esprit routinier du cultivateur, d'ailleurs loin d'être une caractéristique particulière à l'habitant canadien-français, il se serait quand même

${ }^{5}$ La 'Nation canadienne' et l'agriculture (1760-1850) (Trois-Rivières, Éditions du Boréal Express, 1970), 129. C'est en 1947 que le professeur Séguin a soutenu cette thèse de doctorat. 
produit une grave crise agricole, c'est ouvrir des avenues de recherches pour l'exploration et la pondération de divers facteurs objectifs. Ce qu'il importe d'analyser ce sont les faiblesses structurelles de l'économie rurale bas-canadienne qui rendirent la production agricole non concurrentielle face aux multiples contraintes qui l'assaillaient de toutes parts, tant à l'intérieur que de l'extérieur, au point d'en bloquer et d'en paralyser la commercialisation et la modernisation après 1815 . Conséquemment, lorsque «l'enracinement dans la pauvreté» prit des proportions telles qu'on perdit tout espoir d'améliorer les conditions de vie paysanne, il ne resta d'alternative que de se résigner à vivre dans une économie de subsistance précaire ou de chercher une échappatoire à un sort malheureux.

Si le «drame» de la crise agricole occupe une si large place dans $L e$ Bas-Canada c'est qu'il y est traité non seulement en étroite corrélation, mais en véritable symbiose avec la poussée de fièvre nationaliste qui caractérisa la cruciale période de 1815 à 1840 - période à laquelle l'auteur consacre près des deux tiers de son ouvrage. De fait, Fernand Ouellet se montre si manifestement désireux de faire la démonstration de l'hypothèse du "rôle capital» joué par la crise de l'économie rurale dans la «fermentation nationaliste» qu'a cette fin, il a constitué un impressionnant dossier de pièces à conviction.

La «question nationale» constitue le second principal volet du BasCanada. En première partie, l'auteur réserve deux chapitres à «la naissance du nationalisme canadien-français» qu'à l'instar de la crise agricole, il fait remonter au début du dix-neuvième siècle; puis, en seconde et dernière partie, il en analyse la «fermentation» à travers la "géographie des tensions socio-ethniques», l'utilisation par le parti canadien dans sa lutte pour le contrôle des subsides qui aboutit à la «paralysie des institutions politiques», la radicalisation sous l'égide des chefs du mouvement patriote et, enfin, l'exaspération par la rébellion qualifiée de "phénomène révolutionnaire».

Trois principaux facteurs, selon Fernand Ouellet, contribuèrent à la naissance du nationalisme canadien-français: l'avènement d'un mode de gouvernement permettant le développement et l'affrontement de partis politiques dans une arène parlementaire, la formation d'une «nouvelle élite sociale» à la faveur de la montée des professions libérales et du déclin des vieilles familles seigneuriales et la prise de conscience «du caractère presque inexorable» de l'infériorité économique du peuple conquis face à la grandissante domination de la bourgeoisie marchande britannique. Encore fallut-il attendre le tournant du dix-neuvième siècle pour voir se conjuguer ces trois facteurs dans un contexte de «transformations économiques structurelles» qui «secouèrent profondément»la 
société bas-canadienne. C'est «alors seulement» que le terrain se trouva prêt pour l'éclosion et l'épanouissement d'«une idéologie fondée sur le primat de l'ethnicité».

Cette courte présentation suffit à repérer les paramètres dont l'auteur se sert pour situer dans le temps les premières manifestations d'un nationalisme dont la seule forme valable d'expression, selon lui, découlerait du concept de «nation» tel que défini par les théoriciens qui ont inspiré les principes de la Déclaration des Droits de l'Homme de 1789. Se rangeant parmi ceux qui voient dans la Révolution française le grand incubateur du nationalisme européen et occidental, il en tire argument pour nier toute conscience nationale aux seigneurs canadiens qui, une fois devenus conseillers législatifs en vertu de l'Acte de Québec, se proclamèrent les seuls véritables porte-parole de la «Nation Canadienne». $\mathrm{Au}$ dire de l'auteur, ces défenseurs de valeurs monarchiques et aristocratiques furent «incapables d'assumer le sens du mot nation... parce qu'ils étaient tout aussi étrangers à la souveraineté nationale qu'à la souveraineté populaire».

Apparemment fort de cette position de principes, Fernand Ouellet a toujours fermé les yeux sur les nombreux signes de tension ethnique qui, de 1760 jusqu'au tournant du siècle, marquèrent les rapports entre les membres de la bourgeoisie conquérante et les différents porte-parole du peuple conquis. On pourrait mieux comprendre le parti pris de l'auteur à ignorer toute signalisation qui ne réponde pas à ses critères de reconnaissance et à ses normes d'évaluation si sa position, à travers ses écrits, ne paraissait d'une ambivalence réellement trompeuse. D'une part, c'est en faisant appel à une conception rousseauiste du nationalisme qu'il répudie les prétentions des représentants des vieilles familles seigneuriales à parler au nom de la «Nation Canadienne», mais, d'autre part, c'est en invoquant l'importance attachée aux valeurs ethniques qu'il accrédite l'idéologie nationaliste de la "nouvelle élite sociale». De ce jeu sur deux tableaux, l'un pour faire abstraction, avant le tournant du dix-neuvième siècle, des diverses formes d'expression de sentiments ethniques et l'autre, tout au contraire, pour centrer l'attention sur «le primat de l'ethnicité») comme fondement du nationalisme canadien-français, il ressort que ce phénomène apparaît, dès sa naissance, paradoxalement déjà tout armé idéologiquement. Pourtant, à l'époque de la fondation du journal $L e$ Canadien, la «nouvelle élite sociale» était loin d'être prête à assumer «la souveraineté nationale» et encore moins à en partager la notion avec le peuple - si loin, à la vérité, que, pour sauver la position de principes de l'auteur, il faudrait reporter à trois décennies plus tard le baptême, au Bas-Canada, de ce rejeton de la Révolution française.

La continuité historique a plus de consistance que ne le laisse croire le professeur Ouellet sous l'emprise d'un modèle conceptuel qui perturbe sa démarche d'historien. S'il en était moins captif, il pourrait reconnaître 
la justesse de vue de feu Hilda Neatby qui, en conclusion de son ouvrage Quebec: The Revolutionary Age, 1760-1791, avançait l'idée que les seigneurs canadiens, qui se targuaient de leurs titres de "pairs» de la «Nation Canadienne» pour défendre les us et coutumes hérités du Régime français, avaient bel et bien «préparé le terrain» sur lequel la «nouvelle élite sociale» allait trouver le fondement de son idéologie nationaliste en exploitant au maximum le thème des valeurs ethniques léguées par ces descendants de porteurs d'épée et de croix de Saint-Louis. De l'héritage conservateur qu'ils transmirent à la société bas-canadienne, c'est la seule part dont l'auteur fait si peu de cas.

Tout jeune poulain, le nationalisme canadien-français prit rapidement l'allure impétueuse d'un coursier racé destiné aux grands tournois. Quelques années à peine suffirent à l'y préparer dans un Bas-Canada qui, en ce début du dix-neuvième siècle, offrait le spectacle d'une société en pleine "ébullition» où les principaux acteurs fourbissaient leurs armes en vue d'une «lutte sourde, parfois ouverte [et] faite aussi d'alliances temporaires ou partielles». Cette lutte, ayant pour enjeu «le contrôle de la société», mettait aux prises un clergé catholique défendant avec plus de vigueur que jamais le credo d'Ancien Régime, une nouvelle élite de professionnels laïques dont la conscience de classe «débouchait sur une prise de conscience nationale» et une bourgeoisie d'affaires anglophone redevenue «revendicatrice et agressive» sous l'effet des «transformations structurelles» de l'économie coloniale.

Tandis que la traite des pelleteries était en voie de régression, la rapide expansion de l'exploitation forestière et l'essor du commerce du bois contribuaient à renforcer à la fois les assises et l'emprise économiques de la bourgeoisie britannique. Les «marchands aventuriers» angloécossais de la grande épopée de la fourrure durent faire place à de «nouveaux types d'entrepreneurs» qui se montrèrent «beaucoup moins tolérants à l'endroit de l'Ancien Régime social», le considérant comme une entrave majeure à la réalisation de leurs desseins et objectifs capitalistes qui nécessitaient une vaste opération de modernisation (réformes des institutions, établissements bancaires, aménagement du territoire pour fins de transport et de communications, etc...). Malheureusement, observe l'auteur, ce beau "programme de renouveau économique et institutionnel» fut en bonne partie bloqué à l'Assemblée législative, faute d'y gagner l'adhésion du parti canadien.

Pour expliquer ce blocage, l'auteur nous invite (p. 93 et suivantes) à chercher «des raisons à la fois sociales et ethniques» aux "antagonistes les plus profonds et les plus extériorisés» entre les milieux d'affaires anglophones et ceux des professions libérales canadiens-français. Les oppositions étaient d'autant plus irréconciliables que l'enjeu fondamental portait sur «le contrôle de la société» ainsi qu'il se plaît maintenant à l'affirmer, mais sans faire état des travaux de recherches de Gilles Paquet 
et de Jean-Pierre Wallot ${ }^{6}$ qui ont fort bien mis en lumière la véritable dimension de cette lutte débouchant sur le «pouvoir global» de la société. Tout aussi silencieux se montre-t-il quant à l'apport du sociologue Gilles Bourque $^{7}$ dont les hypothèses conduisaient naturellement à la conclusion que chacune des deux formations socio-ethniques rivales cherchait à s'imposer "comme classe sociale dirigeante et comme élite nationale». Mais cette logique découlant des prémisses posées par l'auteur lui-même (p. 113, dernier paragraphe) ne cadre pas avec son schéma interprétatif qui réserve à l'une seulement des parties adverses, les professionnels canadiens-français, de nourrir de semblables ambitions après avoir enfourché la monture du nationalisme. Ainsi, les chefs du parti canadien prennent-ils figure de vindicatifs contestataires du pouvoir économique et politique des anglophones. Se sentant menacées sur leur assise dominante respective, la bourgeoisie et la bureaucratie britanniques prirent conscience du "caractère précaire» de leur situation face aux assauts des représentants de la majorité; elles en éprouvèrent un tel sentiment d' «insécurité» qu'elles développèrent de l'«agressivité»!

Lancé sur la voie de l'approche psychanalytique, Fernand Ouellet s'y laisse entraîner et diagnostique les différentes composantes du corps social. Sa démarche est d'autant plus surprenante qu'on l'en croyait prémuni depuis qu'il avait raillé le schéma d'interprétation freudien de l'école néo-nationaliste de Montréal à propos du «choc» de la Conquête sur «la fragilité émotive» d'une adolescente colonie sevrée de sa «première mère patrie». Que penser de sa propre représentation de la société bas-canadienne des débuts du dix-neuvième siècle? L'auteur n'échappe pas à la tentation d'une dramatisation de la situation par une mise en scène qui invite à croire qu'au sein même de la "nation» conquise, les principaux acteurs étaient en proie à la hantise, à l'obsession ou à l'angoisse, soit de la mort, soit de l'avenir, sous l'effet des grandes secousses qui ébranlaient l'édifice des «équilibres traditionnels» entre les groupes sociaux.

L'élément mystifiant de ce drame social est représenté par la nouvelle élite des professions libérales dont les membres font trembler tout le monde, le gouverneur Craig en tête. Mais, se demande l'auteur, «n'y a-til pas en tout cela un défaut de perception») qui les ferait paraître beaucoup plus terribles qu'ils ne le sont en réalité? Pour en juger, voyons sous quels traits il nous dépeint le parfait représentant de ce milieu professionnel (pp. 108-118).

6 Voir notamment leur ouvrage intitulé: Patronage et Pouvoir dans le Bas-Canada (1794-1812) - un essai d'économie historique (Montréal, Les Presses de l'Université du Québec, 1973).

7 Classes sociales et question nationale au Québec, 1760-1840 (Montréal, Éditions Parti Pris, 1970). 
La formation que ce futur militant a reçue au séminaire ne l'a pas préparé à affronter les problèmes que lui révèle son insertion dans une société en pleine «ébullition» où il se trouve "tiraillé entre différents systèmes de valeurs et sollicité par de multiples allégeances». Impatient et ambitieux de se tailler une place à la faveur du déclin des vieilles familles seigneuriales, il opte pour la carrière politique qui lui apparaît comme «l'instrument de promotion sociale par excellence». Dès son entrée dans l'arène parlementaire, sous la double impulsion de sa conscience de classe et de son idéologie nationaliste, ce militant est lancé dans la lutte contre le pouvoir politique de la bureaucratie et contre la domination économique de la bourgeoisie d'affaires. Mais ce combattant fait piètre figure de «révolutionnaire», car lui aussi, à l'instar de ses adversaires, vit dans la hantise de l'avenir. Ce qui l'inquiète et le trouble surtout, c'est la menace que fait peser sur le sort de la «Nation Canadienne» le programme de renouveau économique et institutionnel qu'entend réaliser la bourgeoisie conquérante pour «l'édification d'une société capitaliste» et britannique ${ }^{8}$. Aussi doit-il mener un combat d'arrière-garde pour sauver du péril la société rurale à laquelle il appartient, de telle sorte que son «idéalisation» des institutions traditionnelles «encourage une réaction contre tout ajustement en profondeur àux iñîériatifs dù monde moderne» (p. 118).

Telle était, selon Fernand Ouellet, «la cause principale du problème canadien-français» que la nouvelle élite sociale chercha à camoufler «en attirant l'attention des milieux populaires sur l'Anglophone». S'il fallait l'en croire il ne s'agirait là que d'une mystification visant à couvrir le refuge des dirigeants du parti canadien dans l'Ancien Régime social. Pour ces «définisseurs du drame» bas-canadien, la présence anglaise était bien plus réelle qu'imaginaire et la situation ne se présentait pas seulement «comme si les Canadiens français étaient systématiquement exclus des hauts paliers de la fonction publique» (p. 97).

Dans son Histoire économique et sociale du Québec, «le premier nationalisme canadien-français» paraissait provenir d'une «angoisse insurmontée face aux exigences d'une adaptation positive à une société individualiste et capitaliste» (p. 211). Si, par "réflexe de peur à l'égard de la conjoncture globale», la nouvelle élite sociale avait opté, «comme il était normal» (!), pour «la préservation de certaines traditions», cependant, «il ne s'agi[ssai]t évidemment pas pour elle de perpétuer tout le passé» (p. 209). Encore fallait-il départager ce qui devait être gardé de ce qui devait être rejeté, compte tenu du «filtrage [qui] s'opère [en elle] en fonction même des prises de conscience et des options présentes) ( $p$. 209). C'était un problème de taille et, à défaut d'avoir pu le résoudre, le professeur Ouellet l'esquive dans Le Bas-Canada.

${ }^{8}$ Ce qu'aurait dû ajouter l'auteur s'il ne se défendait de le reconnaître. 
En dix ans, de 1966 à 1976, de la parution de la première à la seconde magistrale production de Fernand Ouellet, le professionnel canadien-français du tournant du dix-neuvième siècle a si bien intégré sa perception de l'Ancien Régime social qu'il est parvenu à s'en faire une représentation «idéalisée». Remarquons à ce sujet que la prise de conscience historique tout autant que la prise de conscience nationale sont des phénomènes idéologiques incarnés dans l'histoire des peuples et qui répondent à de profonds besoins d'affirmation et d'identification collectives. Il n'appartient donc pas à l'historien de court-circuiter les processus d'évolution nécessaires à leur éclosion. Or, dans la phase d' «effervescence» nationaliste que connut la nouvelle élite sociale, il n'y avait point place pour trouver refuge dans l'«idéalisation» du passé. Cela ne se produisit, ainsi que l'a déjà fort bien exposé le sociologue Fernand Dumont $^{9}$, qu'après l'échec du mouvement insurrectionnel de 1837 qui entraîna "l'effondrement des idéaux» proposés par les chefs du parti patriote. Réduits à l'impuissance, ayant perdu tout espoir de former sur les bords du Saint-Laurent une "Nation Canadienne» indépendante et confrontés à une profonde crise des structures de leur société au moment de l'union des deux Canadas, les contemporains de François-Xavier Garneau éprouveront alors le besoin de valoriser les traditions et, par voie de conséquence, d'idéaliser le passé - ce qui, par la suite, donnera lieu à «une justification théorique (pour la première fois) de la société traditionnelle ${ }^{10}$.

L'image que l'auteur du Bas-Canada nous renvoie des membres des professions libérales est loin d'être aussi «idéalisée» que leur supposée représentation du passé. Qu'on en juge par le portrait qu'au fusain goyesque il nous trace du «premier politicien professionnel», PierreStanislas Bédard (1762-1829), après l'avoir soumis à une véritable autopsie psychanalytique. Il en exhume de tels stigmates de troubles névrotiques et psychotiques que Freud lui-même en serait demeuré perplexe. Ce «maigrichon de petite taille, plutôt laid de figure et de souffreteuse apparence» est un «introverti», un "complexé», un "obséquieux», un "mésadapté dans la société» et un "opprimé dans sa propre maison», éprouvant, avec une "cruelle lucidité», les affres de son "profond sentiment d'infériorité» qui le font vivre dans un «drame permanent». Pour surmonter ou noyer sa «timidité maladive», il doit recourir à l'alcool qui «le délivre de sa paralysie»; mais «ce n'est pas là l'explication de ses performances en tant que politicien», car, malgré «ses faiblesses et ses déficiences», Bédard a connu un «rayonnement énorme». Quelle en serait donc la raison? «Son complexe d'infériorité se doublait d'un complexe de

9 «Idéologie et conscience historique dans la société canadienne-française du XIXe siècle», dans Jean-Paul Bernard, éd., Les idéologies québécoises au 19e siècle (Montréal, Éditions du Boréal Express, 1973), 61-82.

10 Ibid., 77. 
supériorité» (!), grâce auquel il put faire valoir ses remarquables dons intellectuels et s'imposer comme "le chef de file de la classe des professionnels».

Valait-il la peine de descendre si bas pour finalement remonter à la tête? Que cherchait l'auteur dans ces tréfonds? Il n'y avait assurément pas là matière à explication historique, comme il le reconnaît lui-même. A supposer, au mieux, qu'il ait eu l'intention d'indiquer une piste de recherche pour l'interprétation d'un cas pathologique, il paraît l'avoir plutôt embrouillée qu'éclairée. Lorsqu'on est en présence d'une aussi belle tête pensante dont le «leadership» était «surtout intellectuel», de l'aveu même de l'auteur, l'on n'a aucune raison de se perdre dans les méandres d'une fouille pseudo-scientifique. De penseur politique du calibre de Bédard, il s'en trouve si peu dans «notre» histoire nationale qu'à défaut de respect, Fernand Ouellet aurait pu au moins constater que sa mise à nu ne révélait strictement rien de pertinent à la compréhension du «concept de responsabilité ministérielle» mis de l'avant par ce premier chef du parti canadien. Il y avait pourtant là matière à étoffer les quelques pages (135-138) qu'il consacre à ce sujet.

Rattacher la pensée politique de Bédard et de cẹux qui l'entouraient à la tradition de Montesquieu et de Locke pour en déduire que les dirigeants du parti canadien étaient indûment accrochés au passé, c'est faire de l'histoire à rebours. Jamais les auteurs de la Déclaration d'Indépendance et les fondateurs de la République américaine n'auraient imaginé être à contre-courant de l'histoire parce qu'ils se rattachaient à cette tradition. En fait, loin de représenter un recul dans le processus de démocratisation constitutionnelle, la théorie de la responsabilité ministérielle marquait une nette avance sur celle de la séparation des pouvoirs qu'utilisèrent les Pères de la Constitution des États-Unis pour endiguer la poussée démocratique issue de la Révolution américaine. C'est d'ailleurs ce que laisse entendre l'auteur lorsqu'il écrit: «Les adversaires du parti canadien ne tardent pas à comprendre les implications désastreuses pour eux de cette substitution du principe de la primauté de l'Assemblée législative à la théorie de la balance des pouvoirs.» (p. 136).

Malheureusement, sous la contraignante obligation que lui impose son schéma interprétatif, Fernand Ouellet fait subir des contorsions à son analyse historique. Plutôt que d'admettre que les dirigeants du parti canadien réclamaient, en vertu du principe de la responsabilité ministérielle, une extension du contrôle démocratique de la Chambre d'Assemblée, il juge «sans fondement» les accusations de «démocrates» que l'autoritaire gouverneur Craig lança contre eux. La preuve (!) n'en était-elle pas que Bédard, à l'instar de Montesquieu, «cro[ya]it que la démocratie ne saurait être appliquée avec succès dans un grand pays» (p. 133).

S'il est vrai qu'en faisant l'éloge de la Constitution britannique dans son ouvrage De l'Esprit des Lois (1748), le baron de Montesquieu songea 
principalement à défendre les intérêts et les libertés de la classe nobiliaire contre le «despotisme» monarchique, il importe surtout de savoir qu'en prenant parti contre l'absolutisme royal il devint, sans le vouloir, «le héros de tous les adversaires de l'ordre établi» et contribua à «l'ébranlement de cet appareil d'État féodal qui était le seul rempart de la noblesse ${ }^{11}$. Par sa théorie politique du contrepoids ( Il faut que le pouvoir arrête le pouvoir»), Montesquieu donna des armes aux révolutionnaires de 1789 comme en atteste l'article 16 de la Déclaration des Droits de l'Homme. L'Esprit des Lois a donc servi diverses causes et a été l'une des grandes sources d'inspiration des mouvements libéraux nés dans la seconde moitié du dix-huitième siècle. Et c'est précisément dans ce courant libéral que se situaient Bédard et son entourage.

C'est à partir de 1805 - année de l'éclatement de «la querelle des prisons» - que la "crise politique» de la première décennie, «en ses moments d'intensité maximum, mobilise tous les groupes sociaux». Loin d'être «un phénomène passager et superficiel», remarque l'auteur, cette «crise» inaugure une ère de conflits «qui recouvre la première moitié du siècle $»$.

Pour la majorité des historiens, ces affrontements sont purement politiques... Pour d'autres historiens, cette querelle politique se double d'un affrontement ethnique... Le professeur Creighton, pour sa part, y voit une subite aggravation du traditionnel conflit entre l'agriculture et le commerce. Nombre d'historiens décrivent ces luttes comme un épisode dans la bataille pour la survivance ou comme une étape dans l'asservissement des Canadiens français à la «nation Canadian» (p. 119).

Nous voilà donc bien fixés sur quelques-unes des interprétations d'historiens que l'auteur a pris soin d'identifier: «pour la majorité..., pour d'autres... et pour nombre d'autres...». Seuls les professeurs Creighton et Lower méritent signalement; le reste des historiens, canadiens-français pour la plupart, ne vaut même pas la peine d'être distingué. Après tout, quelle importance cela peut-il avoir??? Des compatriotes décédés, dépassés ou déclassés. François-Xavier Garneau, un illustre inconnu, Lionel Groulx, une seule mention dans tout l'ouvrage (p. 112), Maurice Séguin, un épiphénomène, Stanley Bréhaut-Ryerson, un renvoi de justesse en bibliographie avec une mauvaise épellation de son nom... Une exception cependant pour Thomas Chapais, le plus original de tous, treize références. Et Fernand Ouellet, naturellement:

Cette crise nous a paru plus complexe: elle est sans doute politique, à condition qu'on considère la politique comme un niveau où se retrouvent des groupes sociaux et nationaux, des conflits d'intérêts et d'idéologies; comme un niveau où s'expriment des problèmes écono116.

11 Louis Althusser, Montesquieu, la politique et l'histoire (Paris, P.U.F., 1959), 115- 
miques et démographiques et où s'opèrent certaines synthèses ( $p$. 119).

Mais que de frappantes ressemblances dans ce court passage avec le langage auquel nous ont déjà familiarisés les travaux de Gilles Paquet et de Jean-Pierre Wallot ${ }^{12}$ ! Même si l'auteur feint d'ignorer les modèles d'analyse raffinés mis au point par ses collègues, il n'en tire pas moins avantage.

A trop systématiquement exclure les vivants du territoire bascanadien on peut donner l'impression de vouloir l'accaparer pour soi seul. De l'imposante oeuvre de recherche de Jean-Pierre Wallot sur la première décennie du dix-neuvième siècle, l'auteur n'a retenu que le mémoire de maîtrise portant sur «La querelle des prisons au Bas-Canada (1805-1807) ${ }^{13}$. Non content de prendre à partie l'interprétation "purement» nationaliste du jeune maître ès arts d'il y a vingt ans, c'est apparemment en toute bonne conscience qu'il y va de sa petite leçon (p. 126), sans la moindre considération pour l'importante contribution de ce collègue historien depuis sa débutante entreprise de prospection dans le champ de l'histoire. Pourquoi s'encombrer de sa thèse doctorale «Le Bas-Canada sous l'administration de Craig» (Université de Montréal, 1965) lorsqu'on peut s'en remettre à Thomas Chapais pour caractériser l'attitude et le comportement de ce gouverneur (p. 139 et suivantes)? Estce de cette façon que l'auteur tient compte d'une «historiographie en constante évolution»?

L'histoire du Bas-Canada que retrace Fernand Ouellet repose, ainsi qu'il se plaît à le faire croire, uniquement et exclusivement sur sa perception et sa vision du passé. De la «crise politique» initiale déclenchée en 1805 jusqu'à son éclatement final dans les insurrections de 1837-38, on y chercherait en vain une quelconque dimension historiographique. L'auteur y occupe si bien tout le terrain qu'il ne laisse place à aucune autre interprétation que la sienne. Devenu captif du récit ouelletien, le lecteur éprouve l'étrange sensation d'être plongé dans l'absolu de l'histoire. Non seulement n'y trouve-t-il aucune confrontation avec d'autres points de vue, mais aucune remise en question par l'auteur lui-même qui donne l'impression d'être le grand maître de la totalité du savoir historique.

12 Voir, par exemple, «Groupes sociaux et pouvoir: le cas canadien au tournant du XIXe siècle», RHAF, 27, 4 (mars 1974): 558.

13 Ce mémoire de maîtrise a été publié par tranches dans la RHAF, volume XIV, livraisons de juin, septembre, décembre 1960 et mars 1961. 
Dans la seconde et dernière partie du Bas-Canada qui occupe près des deux tiers de l'ouvrage, l'auteur consacre une bonne moitié de son récit au développement de la crise sur le plan politique. Son découpage historique correspond aux principales étapes traditionnellement reconnues et qu'il démarque de la façon suivante: - «le parti canadien et la lutte pour le contrôle des subsides (1815-1828)»; - «la radicalisation du mouvement patriote (1828-1837)»; — «le phénomène révolutionnaire de 1837-38».

C'est sous l'égide de Louis-Joseph Papineau que le parti canadien en vient à «radicaliser» graduellement ses positions et son action au cours de la décennie 1828-1837. La victoire électorale de 1827 avait non seulement consacré le leadership du célèbre chef politique, mais aussi sanctionné la nouvelle appellation de parti patriote. Cependant, ni dans la direction, ni dans l'orientation du parti ne s'était produit de changement en profondeur depuis l'époque de sa formation, dans la première décennie du siècle. D'abord, le parti demeure «l'instrument par lequel le mouvement patriote porte la lutte sociale [i.e. nationale] sur le plan politique» ( $\mathrm{p}$. 294); en second lieu, il ne s'agit pas d'un "parti populaire», mais de «classes moyennes»; troisièmement, le parti continue de lutter «toujours plus âprement» pour la conquête du pouvoir et le contrôle du patronage; et quatrièmement, cette lutte menée au nom du libéralisme et de la démocratie est avant tout vouée à la préservation de l'Ancien Régime social:

La priorité va à la défense des institutions traditionnelles dont le rôle est idéalisé. La canalisation du mécontentement populaire vers des objectifs conservateurs n'est possible que par l'anesthésie plus ou moins complète des aspirations proprement sociales de la masse ( $\mathrm{p}$. 310).

Enfin, cinquièmement, la direction du parti recrute à sa tête des chefs aux troublantes personnalités: "Comme Bédard, L.-J. Papineau appartient à une famille engagée dans le processus de la promotion sociale. De là ce tiraillement perpétuel chez lui entre les valeurs paysannes, aristocratiques et bourgeoises.» (p. 302) Révéré par ses contemporains à la fois comme le "Grand Chef» et «l'homme du peuple», Papineau assigne au parti qu'il dirige «le rôle primordial de défendre ce qu'il considère être l'héritage national des Canadiens français» (p. 307).

Si l'année 1828 marqua le début du processus de «radicalisation» au double plan idéologique et stratégique, point n'était question, nous prévient l'auteur, de rompre «avec les objectifs fondamentaux et permanents» du parti. Cependant, face à «la montée croissante et accélérée du mécontentement populaire», il fallait "une action plus vigoureuse, autrement les chefs risqu[ai]ent d'être débordés par la masse» (p. 329). Les dizaines de milliers de croix inscrites au bas des pétitions qu'emportèrent 
avec eux à Londres les délégués John Neilson, Armand Cuvillier et Denis-Benjamin Viger témoignaient de ce mécontentement. Très longue était la liste des griefs que cette délégation porta à l'attention des autorités métropolitaines et qui fit l'objet du célèbre «Rapport du comité [de la Chambre des Communes] choisi pour s'enquérir sur le gouvernement civil du Canada».

Cette «enquête de 1828» (ainsi que la dénomma Thomas Chapais) se situait à une «importante croisée des chemins» dans les relations entre la métropole et le Bas-Canada ${ }^{14}$. Il valait de traiter sérieusement tant de ce point de vue que de celui de la très malsaine situation qui régnait dans la colonie. L'unique page (327) que l'auteur réserve à cette question et la façon dont il en dispose sont très révélatrices du genre d'analyse historique auquel le confinent ses présupposés idéologiques. Les seuls éléments qui méritent d'être retenus sont ceux qui cadrent avec son «schéma interprétatif». Ainsi ne voit-il dans «les délégués bas-canadiens» que des porte-parole d'un parti à la recherche du pouvoir, des protecteurs de l'Ancien Régime social et des «incriminateurs» de l'administration et de la bureaucratie provinciales. En s'abstenant de faire état des dénonciations du parti patrinte contre les flagrants abus de droit, de pouvoir et de patronage des anglophones du Bas-Canada, Fernand Ouellet a bien suivi l'exemple du secrétaire colonial William Huskisson qui, dans son discours de présentation à la Chambre, prit soin de taire ces aspects les plus compromettants pour la bonne respectabilité anglo-saxonne.

Le professeur Ouellet nourrit de telles préventions contre le parti patriote qu'il a développé des réflexes défensifs à l'égard du parti bureaucrate. Tandis que l'un n'attire dans ses rangs que des gens dévorés par «la faim sans cesse aiguisée et frustrée du pouvoir et du patronage» (p. 330), l'autre ne connaît que des déboires qui le rendent pitoyable aux yeux de l'auteur. Au chapitre X (pp. 387-420), il sympathise avec ces pauvres victimes des victoires électorales des Canadiens et il trouve justification à son apitoiement dans l'analyse faite par le secrétaire de la commission Gosford $^{15}$. Après s'être longuement attardé sur le triste sort du «faible corps» que constituait selon T.F. Elliott, le «parti officiel», c'est presque sur le ton de la désolation que l'auteur en arrive à la conclusion de son chapitre. Mais voici qu'à la toute fin, l'on apprend que ce moribond miné par «trente années de défaites électorales successives... retrouve progressivement son statut de porte-parole des anglophones après 1830».

14 Voir à ce sujet, Peter Burroughs, The Canadian Crisis and British Colonial Policy, 1828-1841 (Londres, 1972).

15 Selon T.F. Elliott, les «deux factions» constituantes du parti bureaucratique étaient représentées d'une part, par le «parti officiel» qui regroupait une coterie de privilégiés occupant "les plus hauts emplois», mais "dénués [en fait] de tous les éléments réels du pouvoir» et, d'autre part, par le «parti anglais» qui recrutait les éléments les plus dynamiques du monde anglophone. 
Valait-il la peine de suivre un si long cheminement pour finalement reconnaître que «la force véritable du parti bureaucrate réside dans les liens qui l'unissent aux détenteurs du pouvoir économique» (p. 420)?

Convaincu que la position socio-économique dominante de la bourgeoisie anglo-saxonne tenait davantage au dynamisme et à l'esprit d'entreprise capitaliste de ses gros brasseurs d'affaires qu'aux faveurs et au patronage du régime britannique, l'auteur parvient difficilement à comprendre pourquoi les chefs patriotes n'ont pas su ou voulu reconnaître ce que lui-même considère comme «la réalité». Et, à défaut de compréhension, il taxe de «faiblesse majeure» la propension des dirigeants du mouvement patriote à «toujours attribuer des causes politiques aux phénomènes économiques et sociaux» (p. 388).

Lorsque Papineau clamait: «Vous voulez tout avoir: le pouvoir, les places et l'or", il s'en prenait tout autant au système oligarchique qu'à la volonté de domination des anglophones, englobant dans cette dénonciation les «deux factions» constituantes du parti bureaucrate que distinguait T.F. Elliott pour fin d'analyse. De ces deux factions, remarquait ce dernier, le «parti anglais», par opposition au «parti [bureaucrate] officiel», manifestait le plus d'«ambition de dominer». Et le secrétaire de la commission Gosford observait: «Les Canadiens français ne sauraient guère manquer de s'apercevoir que les Anglais se sont emparés de toutes les richesses ainsi que du pouvoir dans chaque pays où ils ont pu prendre pied.» (RAC (1883): 168.) N'était-ce pas ce qui, fondamentalement, rendait jaloux les premiers si «inquiets et jaloux»?

Mais le parti pris de l'auteur est tel que, fermant les yeux et gardant le silence sur le comportement et les agissements de l'élite dominante et des colonisateurs britanniques, il ne décèle, découvre et dévoile de fautes que chez les patriotes canadiens - ces irréductibles contestataires du pouvoir colonial établi. Et plus encore que leur contestation, il réprouve l'insatiable avidité qui, selon lui, motiverait l'action des dirigeants du parti patriote. À l'en croire, le projet politique du «Grand Chef», au risque même de devenir suicidaire, "est de faire passer la totalité du pouvoir dans les mains des classes moyennes canadiennes-françaises» (p. 347). Et c'est dans le but d'atteindre cet objectif suprême que Papineau engage résolument ses troupes dans la voie de la radicalisation, au double plan idéologique et stratégique.

Envisagée dans cette perspective, la lutte nationaliste prend l'allure d'un véritable combat où l'arsenal idéologique sert à des fins stratégiques. Aussi le lecteur ne se surprendra-t-il pas trop de lire que le mouvement patriote, une fois engagé dans ce processus de radicalisation, passe rapidement «d'une idéologie nationaliste, portant le masque libéral et réformiste, à un nationalisme enrobé d'idées démocratiques et républicaines» (p. 347). Papineau donne l'impression de brûler les étapes de telle 
sorte que sa «stratégie qui implique l'extension du principe électif à tous les niveaux où s'exerce le pouvoir» le conduit, vers 1830 , «à une remise en question du système colonial et des institutions britanniques». Et pour mieux «fonder son action sur des principes qui la justifient pleinement», il recourt alors aux théories rousseauistes et aux conceptions jeffersonienne et jacksonienne de la démocratie américaine (pp. 334-336).

Loin de prendre au sérieux cette «reconstruction idéologique», l'auteur n'hésite pas à qualifier d'«exercice intellectuel» démagogique la «mythologie» invoquée par Papineau pour «démontrer la contradiction fondamentale vécue par la nation canadienne-française tiraillée entre un état social soi-disant démocratique et un régime politique à caractère monarchique et aristocratique imposé par l'Angleterre» (p. 339). S'il fallait l'en croire, les arguments que ne cesse de brandir dans ses discours le seigneur de la Petite-Nation ne répondraient qu'aux «exigences de l'action» commandant l'utilisation à fond de la stratégie de «l'homme du peuple». À ce compte, que penser des conceptions démocratiques et républicaines du maître inspirateur de Papineau, Thomas Jefferson, qui fut membre de l'aristocratie terrienne de la Virginie et dont le vaste domaine de Monticello valait bien celui de la seigneurie de Montebello?

Que l'on compare avec le portrait qu'en 1960, Fernand Ouellet nous brossait de Louis-Joseph Papineau dans une publication de la Société historique du Canada. Ne partageait-il pas alors la «mythologie» de cet "être divisé» entre son conservatisme social et son libéralisme politique? Qu'est devenu pour l'auteur du Bas-Canada ce «héraut de la démocratie [qui] se doublait d'un héros de l'indépendance nationale»? - Un fieffé démagogue doublé d'un stratège consommé qui ne cesse d'occulter ses intérêts de classe et sa soif du pouvoir derrière le masque du libéralisme démocratique et républicain. Non satisfait d'avoir bafoué PierreStanislas Bédard, le premier porte-parole «professionnel» du nationalisme canadien-français, le professeur Ouellet passe aussi au crible de sa systématique entreprise démystificatrice les convictions du «Grand Chef» qu'il qualifiait jadis de «démocrate ardent» et en qui il trouvait «une résonnance réelle» des courants de pensée libérale de l'époque. Le cynisme iconoclaste dont il fait maintenant preuve le conduit, à son insu, à une «reconstruction» stratégique du mouvement patriote qui aboutit à une mise en scène «révolutionnaire» comportant, elle aussi, sa part de «mythologie» et de mystification.

Naturellement, toute stratégie révolutionnaire digne de ce nom doit se préparer dans le secret et selon un plan d'action préalablement établi. Il faut également disposer d'une organisation efficace et bien structurée. Tous ces principaux éléments se retrouvent dans le scénario du professeur Ouellet et son montage, à vrai dire, est si bien réussi qu'il crée l'impression du réel. "Dirigé par un chef éclatant, autoritaire, bâtisseur d'idéologie, créateur de mythes et de symboles, le parti patriote est servi 
par une organisation complexe, hiérarchisée et active dans les moindres localités.» (p. 358).

Dès ses premiers écrits sur Papineau, l'auteur avait pris la contrepartie de la thèse de la «non-préméditation» et de la «non-préparation» originellement formulée par le «Grand Chef» lui-même dans son Histoire de l'Insurrection du Canada (1839): «Je mets le gouvernement anglais au défi de me démentir, quand j'affirme qu'aucun de nous n'avait préparé, voulu ou même prévu, la résistance armée.» Cette thèse, qui avait pour principal corollaire la «provocation» gouvernementale, fut non seulement défendue par les principaux collaborateurs de Papineau, tels son adjoint E.B. O'Callaghan et le docteur Wolfred Nelson, mais endossée par de nombreux contemporains avant d'être reprise dans l'historiographie sous la plume notamment de L.-O. David, Gérard Filteau et Lionel Groulx.

À l'appui de son antithèse, Fernand Ouellet dispose d'un arsenal d'arguments, de faits et de preuves trop compromettants pour qu'on ne puisse jamais plus écrire et encore moins soutenir à l'instar de Groulx que le soulèvement de ' 37 fut «un mouvement improvisé et presque spontané en ses sursauts violents» ${ }^{16}$. Faut-il croire pour autant que tout avait été planifié et orchestré d'avance? «Incontestablement, affirme l'auteur, les événements se déroulent selon un plan pré-établi» qui, enchaîne-t-il, prévoyait «deux phases»: la première, «l'agitation soi-disant légale»; la seconde, l'insurrection armée dont «le moment choisi... se situe[rait] après 'la prise des glaces' à la suite d'une convention générale prévue pour le 4 décembre, devant donner lieu à une déclaration d'indépendance et à l'établissement d'un gouvernement provisoire» (p. 434).

Pour séduisante qu'elle soit, cette «reconstruction» stratégique comporte le grave inconvénient de ne pas dissocier ce qui fut vécu dans l'imaginaire de ce qui fut perçu comme réalisable tant sur le plan tactique que sur celui des objectifs. C'est évidemment dans l'ordre du rêve que se situe, par exemple, le témoignage du docteur Timothée Kimber, de Chambly, qui prétendait, au lendemain de la dernière grande assemblée - celle des «Six Comtés» tenue à Saint-Charles le 23 octobre 1837 -: «Du moment que la rivière [le Saint-Laurent] sera prise, nous irons avec 40 ou 50,000 hommes armés prendre Montréal; tous les habitants sont bien armés et bien fournis de munitions et bien déterminés et, après Montréal, nous prendrons Québec.» (p. 446). Qu'en était-il en réalité? Si l'on en juge par l'effet désorganisateur de l'intervention gouvernementale de la mi-novembre (alors que les autorités coloniales lancèrent des mandats d'arrestation contre les dirigeants du mouvement), on peut se questionner sérieusement sur la supposée stratégie révolutionnaire des patriotes.

16 Histoire du Canada français depuis la découverte (Montréal, 4e édition, 2 tomes, 1962), II: 169. 
Après réflexion sur l'échec du soulèvement de 1837, l'auteur lui-même en arrive à conclure: "Même si l'insurrection avait éclaté au moment prévu, le 4 décembre, il n'est pas du tout certain que l'organisation aurait été au point.» (p. 466). Assurément non, peut-on penser, puisque la décision de Gosford de faire arrêter les chefs «prend les patriotes au dépourvu et jette la consternation dans leurs rangs» (p. 458).

Cette réalité ne témoigne-t-elle pas d'une absence ou, à tout le moins, d'un manque de stratégie révolutionnaire et n'y aurait-il pas lieu, à partir de là, de revoir le scénario de l'auteur? L'histoire commencerait ainsi: le quinze novembre 1837, le «Grand Chef» et son adjoint O'Callaghan, après avoir fui Montréal, trouvèrent refuge à Varennes où, avec quelques autres patriotes, ils tinrent conciliabule pour déterminer un plan d'action face à la répression gouvernementale. Un témoin, du nom d'Amury Girod, rapporta ce qui suit de la scène:

Je ne me rappelle pas quel est celui d'entre nous... qui proposa qu'on convoquât une convention et qu'on établît un gouvernement provisoire. Nous acquiescâmes à sa proposition, mais nous ajoutâmes que cette première mesure équivalait à un acte de rébellion ouverte et gu'til serait bon de chercher les moyens d'organiser le peunle et de se procurer des armes et des munitions... (RAC (1923): 408-409).

Est-ce la suite de cette histoire qui inspira au professeur Ouellet l'article paru en 1968 sous le titre: «Les insurrections de 1837-38: un phénomène social» ${ }^{17}$ ? Chose certaine, il n'était point question alors d'y voir un mouvement révolutionnaire stratégiquement organisé par la haute direction du parti:

En fait les insurrections [de 1837-38]... offrent un spectacle d'anarchie, d'impulsivité mal canalisée et d'une sorte d'impuissance à agir avec efficacité... Ces énormes rassemblements de paysans... étaient plutôt le fruit de l'instinct grégaire que d'une véritable stratégie... Il semble que le comportement des milieux populaires au moment de l'action reflète la déficience du leadership à tous les niveaux... Papineau qui incarnait le mouvement... se complut dans l'ambiguïté et il se révéla inapte à l'action... Au point de départ, tout effort sérieux d'organisation se trouvait bloqué... (Éléments d'histoire sociale, 374-375).

Le Bas-Canada comporte une matière trop abondante et trop riche pour prétendre en avoir épuisé la substance. Il est au moins deux autres aspects sur lesquels j'aurais aimé m'étendre: d'une part, la représentation ouelletienne de la stratégie cléricale de Monseigneur Lartigue face à

17 Histoire sociale - Social History, no 2 (novembre 1968): 54-82; reproduit dans Eléments d'histoire sociale du Bas-Canada (Montréal, HMH, 1972), 351-379. 
la radicalisation du mouvement nationaliste et, d'autre part, l'utilisation plutôt débridée que l'auteur fait des «Poll Books» dans le but d' "explorer», ainsi qu'il le précise dans sa préface, «les rapports entre les mouvements politiques, les changements socio-ethniques, les problèmes économiques et démographiques». On y trouve une bonne illustration du genre d'exploration «scientifique» que Fernand Ouellet affectionne tout particulièrement effectuer grâce à la magie des chiffres. Aussi ne doit-on pas s'étonner de le voir, avec ses «Poll Books», s'aventurer à sonder les «motivations» de l'électorat paysan et à détecter dans les communautés rurales les «rivalités entre paroisses et clans familiaux» (pp. 366-368).

D'un auteur qui attache une si grande importance à la quantification des données, on s'attendait à ce qu'il soigne la présentation des très nombreux tableaux statistiques figurant dans son ouvrage. Malheureusement, comme le fait remarquer Nicole Gagnon, du département de sociologie de l'Université Laval, ces tableaux «manquent en général d'intelligibilité visuelle» ${ }^{18}$. La lecture des pourcentages qui accompagnent les données impose trop souvent une recherche des coordonnées pour en déterminer la distribution verticale ou horizontale. Dans certains cas, notamment pour l'interprétation des résultats électoraux, il faut faire preuve d'imagination ou d'ingéniosité, à défaut d'avoir reçu une initiation au système électoral de l'époque (exemple: le tableau du haut de la page 369). Les pourcentages produits dans le commentaire qui accompagne le tableau du haut de la page 360 n'ont aucun rapport avec ceux du tableau lui-même. Faute de savoir qu'il y eut d'autres candidats en liste pour se partager les voix des Canadiens français, les non-initiés comprendront difficilement que $43 \%$ représentait un «appui massif» alors que, d'après le tableau, John Neilson avait obtenu au «total» $84 \%$ des suffrages des francophones. Par ailleurs, on s'explique mal que $84 \%$ des votes obtenus par G. Desbarats lui soient venus des paroisses de Sainte-Claire et de Frampton (comté de Beauce) alors que, pour ces deux dernières, le tableau de la page 370 n'indique que 108 voix (5 et 103) sur un «total» de 410.

Il serait ici fastidieux de relever toutes les coquilles (inversions de lettres et de chiffres) qui parsèment le texte. On aurait au moins espéré un bon nettoyage à l'occasion de la «seconde édition» (janvier 1980) qui, en fait, n'est qu'un nouveau tirage de la version originale. Il n'y a donc pas à se surprendre d'y retrouver le même lapsus que dans la première édition: Pierre-Antoine Panet au lieu de Jean-Antoine Panet indiqué comme candidat à la présidence de la première Chambre d'Assemblée du Bas-Canada (page 74, 4e ligne). Le lapsus est d'autant plus frappant que son cousin, Pierre-Louis Panet, fut l'un des deux seuls Canadiens français à voter contre cette nomination.

18 Compte rendu critique paru dans Recherches sociographiques, XIX, 3 (sept.-déc. 1978): 408-411. 
Aux yeux de la sociologue Nicole Gagnon, il apparaît «tout à fait secondaire» que Fernand Ouellet «s'encombre peu de précision démonstrative» et que, «mal initié peut-être au b-a ba de l'analyse multivariée, il semble tout aussi bien prendre ses aises avec les principes élémentaires de la critique historique $>{ }^{19}$. L'historien que je suis ne peut traiter aussi à la légère de tels manquements aux exigences méthodologiques du métier que pratique l'auteur. Si valable que soit «la reconstruction du processus historique» dans le cadre d'un "schéma interprétatif» elle ne saurait cependant être faite au détriment du souci d'objectivité qui doit constituer la préoccupation majeure de l'historien dans sa recherche d'une adéquation de l'analyse à une réalité toujours complexe et multiforme, soumise aux multiples variables de la conjoncture et aux changements structurels, et à jamais irréductible aux procédés de simplification.

Au terme du récit ouelletien, le lecteur refermera le livre en songeant que, malgré tout ce qu'on peut trouver à redire, l'auteur a l'insigne mérite d'avoir rendu l'histoire du Bas-Canada non seulement intéressante, mais captivante grâce à l'éclairage d'une formalisation théorique qui, pour reprendre l'heureuse expression de Nicole Gagnon, introduit "une intelligihilité vivante dans la reconstruction du processus historique» ${ }^{20}$. À ce point de vue, le professeur Ouellet a assurément donné le meilleur de lui-même et tous ses lecteurs lui sauront gré d'avoir mis au service de l'histoire son inlassable labeur de chercheur passionné. 